\title{
A Preliminary Study on a Newly Developed Cell-hybridized Artificial Bone in a Beagle Dog: Bone Marrow Stromal Cells with an Interconnected Porous Hydroxyapatite Scaffold
}

Kazuya Doi, DDS, PhD, Takayasu Kubo, DDS, PhD, Kazuhiko Hayashi DDS, PhD, and Yasumasa Akagawa, DDS, PhD

Department of Advanced Prosthodontics, Division of Cervico-Gnathostomatology, Programs for Applied Biomedicine, Hiroshima University Graduate School of Biomedical Sciences, Hiroshima, Japan

\section{Clinical significance}

Tissue engineering has attracted much attention to solve the disadvantages associated with bone grafts. Bone regeneration with bone marrow stromal cells (BMSCs) requires an optimal scaffold and interconnected porous hydroxyapatite has recently been developed as a possible candidate. This study was designed to clarify a possibility of the application of a hybrid artificial bone and the results obtained may be the possible use of cell-hybridized artificial bone.

\begin{abstract}
Purpose: This study was performed to clarify the possibility of bone regeneration with a cell-hybridized artificial bone by evaluating bone formation in the beagle dog femur.

Methods: Bone marrow stromal cells (BMSCs) /interconnected porous hydroxyapatite (IP-CHA) composites as a cell-hybridized artificial bone were made by the injection of the BMSCs solution into IP-CHA. The distribution of BMSCs in IP-CHA was examined by scanning electron microscopy. Two BMSCs/IP-CHA composites (the BMSCs of one composite were labeled fluorescence) were placed into each of two bone sockets $(\phi 3.7 \mathrm{~mm} \times 7 \mathrm{~mm})$ prepared in the right femur of the beagle dog. After 4 weeks of surgery, new bone formation in the socket was examined by light microscopy and fluorescence microscopy.

Results: SEM showed well distribution of the injected BMSCs in the pores of IP-CHA. At 4 weeks, new bone formation was detected at the cortical areas of the composites, and injected BMSCs were identified in the new bone area of the interconnected pores.
\end{abstract}

Corresponding to: Dr Kazuya Doi

Department of Advanced Prosthodontics, Division of

Cervico-Gnathostomatology, Programs for Applied

Biomedicine, Hiroshima University Graduate School of

Biomedical Sciences,

1-2-3 Kasumi, Minami-ku, Hiroshima 734-8553, Japan

Tel: +81-82-257-5677, Fax: +81-82-257-5679

E-mail: kazuya17@hiroshima-u.ac.jp

Received on August 11, 2006 / Accepted on September 12, 2006
Conclusion: The limited results of this study may suggest that BMSCs/IP-CHA composites as cell-hybridized artificial bone is a possible candidate for bone regeneration.

Key words: interconnected porous hydroxyapatite, bone regeneration, cell-hybridized artificial bone

\section{Introduction}

Bone harvested from the mandible and ilium has been widely used as autogenous bone grafts in clinical situations requiring bone grafting in the oral cavity. ${ }^{1-3}$ However, problems still exist including the limited volume of harvested bone available and complications at the donor site as a result of the necessary surgical procedure..$^{4,5}$ To overcome these problems and to obtain predictable bone grafting, other graft materials have been developed for use as bone substitutes. Hydroxyapatite (HA) has been widely used as a graft material with excellent biocompatibility and direct bonding to repaired bone tissue..$^{6-9}$ However, this direct bonding has been limited to the surface of the HA. Recently, a newly developed HA with an interconnected porous structure (IP-CHA) has been introduced and has been demonstrated to have substantial porosity. ${ }^{10} \mathrm{Be}^{-}$ cause IP-CHA has a systematic arrangement of uniform spherical interconnected pores, this material may possess qualities suitable for a graft material with osteoconduction, allowing cells to penetrate into the IP-CHA. It is well known that bone marrow stromal cells (BMSCs) contain progenitors capable of differentiation into such mesenchymal lineages as bone, cartilage, fat and connective tissue. ${ }^{11,12}$ Some studies have shown favorable bone formation by BMSCs on scaffolds with a coculture. ${ }^{13,14}$ In fact, IP-CHA has recently 


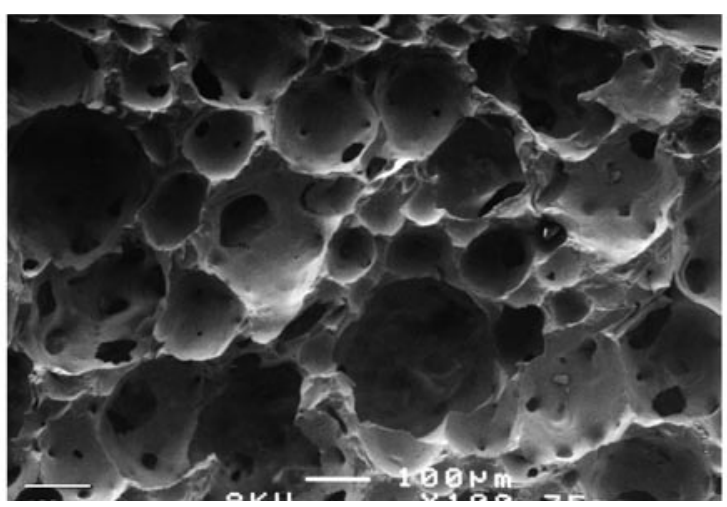

Fig. 1 SEM image of IP-CHA. A uniform size of pores can be seen, and almost all pores were connected through interconnective pores.

been used as a scaffold with BMSCs without co- $^{-}$ culture and a study using Sprague-Dawley rats reported excellent bone formation. ${ }^{15}$ However, there is a lack of studies using large animals. Such studies are needed to explore the possibility of a hybrid artificial bone as graft material (BMSCs/IP-CHA) using BMSCs on a scaffold of IPCHA.

This study was, therefore, performed to clarify the possibility of bone regeneration with BMSCs/ IP-CHA composites by evaluating bone formation in the beagle dog femur.

\section{Materials and methods}

\section{Animal}

After receiving approval from the Research Facilities Committee for Laboratory Animal Science, Hiroshima University School of Medicine, one beagle dog (12 kg in weight and aged 14 months) was used in this study.

\section{Scaffold}

IP-CHA (NEOBONE ${ }^{\circledR}$, Toshiba ceramics Co., Tokyo, Japan) with a $75 \%$ porosity, a mean pore diameter of $150 \mu \mathrm{m}$ and interconnective pore diameter of $40 \mu \mathrm{m}$ (Fig. 1) was used as a scaffold. Both two disc-type IP-CHAs (diameter $20 \mathrm{~mm}$, height $6 \mathrm{~mm}$ ) and two cylinder-types (diameter $3.7 \mathrm{~mm}$, height $7 \mathrm{~mm}$ ) were prepared as specimens.

\section{BMSCs harvests and culture}

BMSCs were harvested by a $5 \mathrm{ml}$ aspiration of iliac bone marrow from the dog. Cell culture was performed in accordance with the technique described by Tsutsumi et al. ${ }^{16}$ BMSCs were seeded in $100 \mathrm{~mm}$ cell culture dishes and maintained in $10 \mathrm{ml}$ of Dulbecco's modified Eagle's medium (Sigma, USA) supplemented with 10\% fetal bovine serum, $0.05 \mathrm{unit} / \mathrm{ml}$ penicillin, and 0.05 $\mathrm{mg} / \mathrm{ml}$ streptomycin (Gibco, USA). After three days of seeding, floating cells were removed and the medium was replaced with fresh medium. Passages were performed when cells were approaching confluence. Then, cells were seeded at $5 \times 10^{3}$ cell $/ \mathrm{cm}^{2}$ in $10 \mathrm{~mm}$ dishes and maintained in a medium containing $3 \mathrm{ng} / \mathrm{ml}$ fibroblast growth factor-2 for 2 weeks. A number of BMSCs were observed in these adherent cells. The cells were harvested with trypsin plus EDTA, washed with phosphate buffered saline, and stored in liquid nitrogen until use. In this study, cell passage 4 was used.

\section{BMSCs labeling and sample preparation}

BMSCs were labeled with the red fluorescent dye PKH-26 (Sigma, UK) before transplantation. These BMSCs were mixed with medium and 1 $\mathrm{ml}$ of BMSC solution (cell density $1.0 \times 10^{6}$ ) was injected into the IP-CHA scaffold. Through this method, both disc-type and cylinder-type BMSCs/ IP-CHA composites were fabricated with the use of disc-type and cylinder-type IP-CHA.

\section{Scanning electron microscopy (SEM) evaluation of BMSCs/IP-CHA}

Disc-type BMSCs/IP-CHA composites were examined with SEM to evaluate cell penetration into the deep pores. Twenty-four hours after injection of the BMSCs, the BMSCs/IP-CHA composites were fixed and treated for SEM observation. The sample was cut in half at the central part and this area was examined.

\section{Animal experiment}

Under general anesthesia (sodium pentobarbital, $40 \mathrm{mg} / \mathrm{kg})$ and local infiltrated anesthesia (2\% lidocaine with 1:80,000 noradrenaline), two bone sockets of $3.7 \mathrm{~mm}$ diameter and $7 \mathrm{~mm}$ depth were prepared surgically with $3.7 \mathrm{~mm}$ drills on the right femur of the animal for BMSCs/IP-CHA composite placement. Cylinder-type BMSCs/IPCHA composites were placed into the bone sockets (Fig. 2). Four weeks after surgery, the animal was sacrificed under general anesthesia, the femur was dissected, and tissue blocks containing BMSCs/IP-CHA were obtained.

One of the two tissue blocks from the right femur was fixed in 10\% neutral formalin. After 


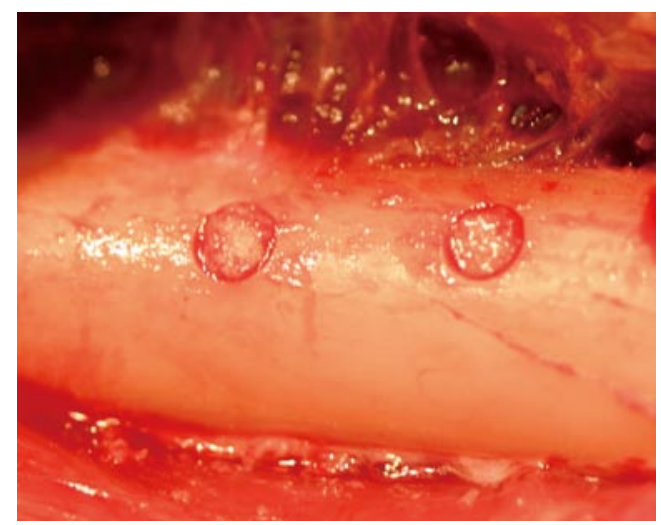

Fig. 2 Two BMSCs/IP-CHA structures are placed into the bone sockets in the right femur.
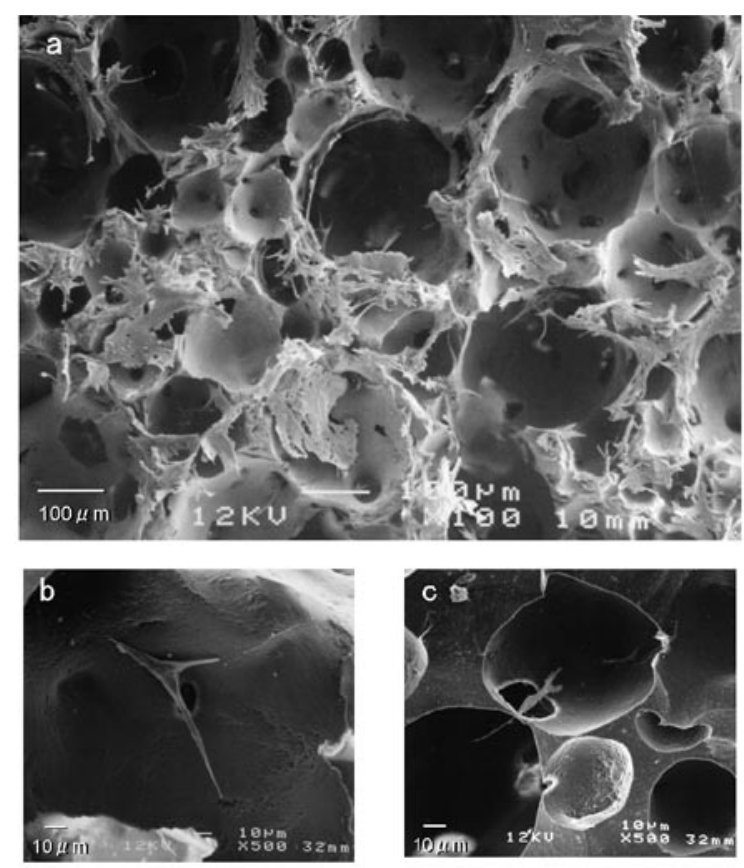

Fig. 3 SEM image of BMSCs/IP-CHA composites. a: Injected BMSCs are distributed extensively, not only on the surface but also in the deep interpore area. b: Injected BMSCs are attached to the walls of the pores. c: Injected BMSCs penetrates through interconnected pores.

decalcification in hydrochloride for 5 days, the tissue block was dehydrated through graded ethanol, cleared with xylene, embedded in paraffin, cut into $5 \mu \mathrm{m}$ thick sections with a microtome and the sections were stained with hematoxylin and eosin (H-E). The sample was examined histologically with a light microscope. The second tissue block was frozen with sucrose using dry ice with hexane and cut into the central part of the graft material. The sample was cut into $5 \mu \mathrm{m}^{-}$ thick sections with a cryostat microtome (Leica, Germany) at $-25^{\circ} \mathrm{C}$ and unstained specimens were examined with fluorescent microscopy.
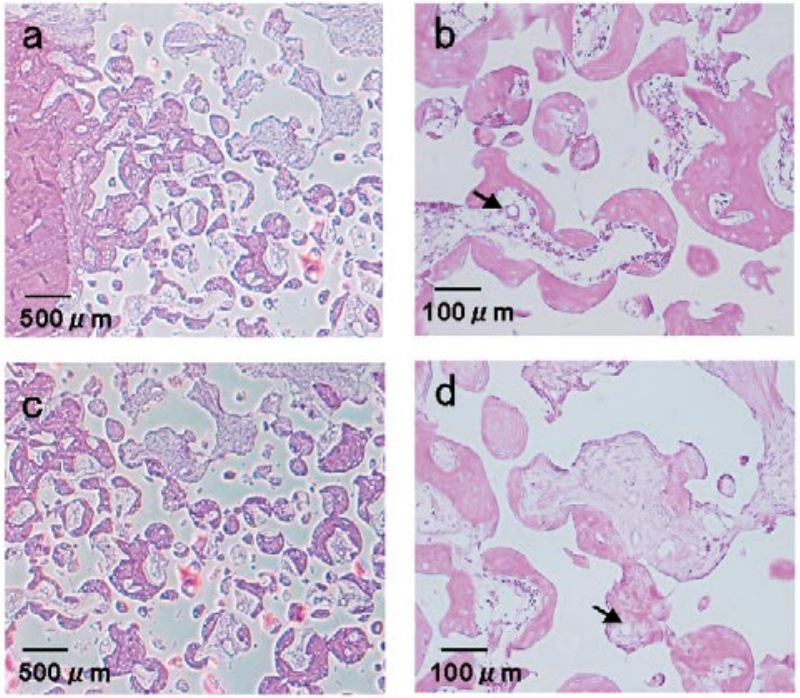

Fig. 4 Histological specimen at the cortical bone area at 4 weeks after placement, arrows: show the vascular formation. $a, b$ : At the marginal area, most pores are filled with regenerated bone. c,d: At the central area, the pores are filled with woven bone, lamella bone and connective tissue with vascular formation.

\section{Results}

\section{SEM evaluation of BMSCs/IP-CHA}

Injected BMSCs extended to the deep part of IP-CHA and they were visible not only on the surface of IP-CHA but also in the interconnected interior pores at the deep area. Injected BMSCs had expanded well and attached to the walls of the pores (Fig. 3). At $3 \mathrm{~mm}$ below the surface, injected BMSCs were still found in the pores.

\section{Histological evaluation of animal experiment}

At 4 weeks after surgery, new bone formation was observed in the BMSCs/IP-CHA placed in the bone sockets. Newly formed bone appeared to be prominent in the cortical bone area. Most pores were filled with woven bone, lamella bone and connective tissue with vascular formation (Fig. 4).

Fluorescent-labeled BMSCs were observed in the BMSCs/IP-CHA. Fluorescent-labeled BMSCs were identified in the interconnected pores, where new bone formation was detected (Fig. 5 $a, b, c, d)$. 

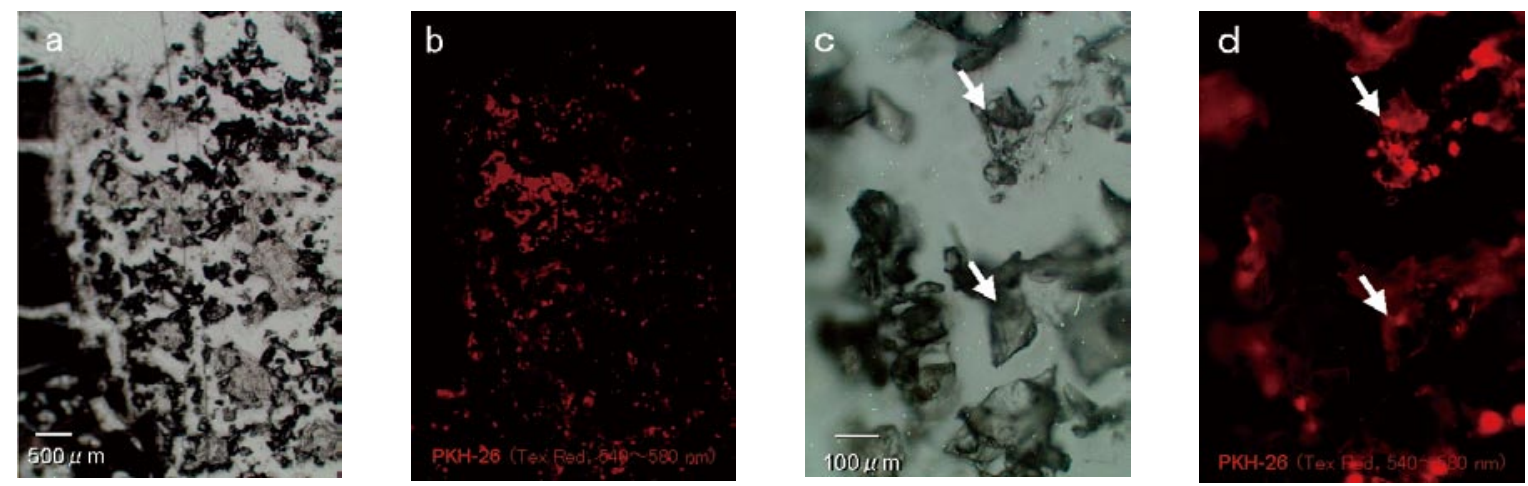

Fig. 5 Fluorescence specimen at the cortical bone area at 4 weeks after placement. a,b: Fluorescent-labeled BMSCs are recognized in the BMSCs/IP- CHA composites, although they are not observed in the host tissues. c,d: Fluorescent-labeled BMSCs are identified in the pores, where new bone formation is detected. Arrows indicates same points.

\section{Discussion}

The limited and preliminary results of this study suggest that injected BMSCs may promote bone regeneration in the pores of IP-CHA. It has been reported that BMSCs can be induced to differentiate into osteoblasts, chondrocytes and adipocytes and that this differentiation is affected by the environments of the surrounding tissue. ${ }^{17-19}$ By transplanting BMSCs with an IP-CHA scaffold, promotion of tissue regeneration may be achieved. HA has been widely used as a bone substitute. Recently, HA used as a scaffold for tissue engineering has demonstrated good bone formation. ${ }^{6-9}$ However, because of inadequate pore connections in conventional HA, osteoconduction has been limited to the surface. ${ }^{20}$ Generally speaking, over $10 \mu \mathrm{m}$ in diameter will permit osteoconduction, ${ }^{21}$ because the size of the nucleus in most mammalian cells is over $10 \mu \mathrm{m}$. It has been reported that bone ingrowth penetrating into the HA was no more than $300 \mu \mathrm{m}$ from the interface at 4 months after implantation in humans. ${ }^{22}$ In the study reported here, based on histological evaluation, the interior of the placed BMSCs/IP-CHA was filled with new bone, connective tissue and vascularization. The IP-CHA used in this study had a systematic arrangement of uniform pores, and almost all pores were interconnected. Thus, it is suggested that good osteoconduction was achieved. Furthermore, this methodology also has the advantage of the cells or growth factors being hybridized, not only on the surface but also in the IP-CHA interior. In this study, injected BMSCs were observed in the interior of the IP-CHA. The injected cells were considered to have reached the deep part of the material. Prior to this study, it could be concluded that injected cells at the interior of IP-CHA may no longer be alive because of the shortage of oxygen or nutrition supply. However, fluorescence observation demonstrated that fluorescentlabeled cells were well distributed in the broad area of IP-CHA, suggesting that the injected cells in the pores were alive. BMSCs are adhesive cells and adhere to the surface of pores if injected into IP-CHA. However, when the IP-CHA is placed within an organism, adhered cells may flow via the blood stream and outflow to other areas. In addition, the defects may be filled with bone marrow, which may mean that bone formation was achieved by BMSCs originating from the animal's own bone marrow. However, it was shown that the injected cells were widely distributed and some of them were recognized in IP-CHA as fluorescent-labeled cells. This may mean that the injected cells were at least involved in bone tissue formation in the pores. Our previous study in the laboratory showed bone formation promoted by BMSCs/IP-CHA rather than only IP-CHA at early stage of 4 weeks after placement in a beagle dog. It may be possible to use BMSCs/IPCHA in the clinical environment, however, there is a problem of bovine serum in the cell culture medium which may be associated with the risk of bovine spongiform encephalopathy or other unknown infections. To solve this problem, a culture method using serum free medium or autologous serum may be recommended.

The findings of this limited study suggest that IP-CHA may be used to hybridize BMSCs and that injected BMSCs/IP-CHA could enhance bone regeneration. It is anticipated that further study will more fully clarify the acceleration of bone 
regeneration with the use of osteogenic differentiated BMSCs-hybridized artificial bone with an IP-CHA scaffold.

Acknowledgements: This study was supported by a Grant-in-aid for Scientific Research (No.16659536) from Japan Society for the Promotion of Science.

\section{References}

1. Schallhorn RG. Long term evaluation of osseous grafts in periodontal therapy. Int Dent J 30: 101-116, 1980.

2. Boyne PJ. Advances in preprosthetic surgery and implantation. Curr Opin Dent 1: 277-281, 1991.

3. Becker W, Urist M, Becker BE et al. Clinical and histologic observations of sites implanted with intraoral autologous bone grafts or allografts. 15 human case reports. J Periodonto 67: 1025-1033, 1996.

4. Burchardt H. The biology of bone graft repair. Clin Orthop Relat Res 174: 28-42, 1983.

5. Reuben SS, Vieira P, Faruqi S et al. Local administration of morphine for analgesia after iliac bone graft harvest. Anesthesiol 95: 390-394, 2001.

6. Yoshikawa T, Ohgushi H, Tamai S. Immediate bone forming capability of prefabricated osteogenic hydroxyapatite. J Biomed Mater Res 32: 481-492, 1996.

7. Yoshikawa T, Ohgushi H, Dohi Y et al. Viable bone formation porous hydroxyapatite: marrow cellderived in vitro bone on the surface of ceramics. Biomed Mater Eng 7: 49-58, 1997.

8. Nordstrom E, Ohgushi H, Yoshikawa T et al. Osteogenic differentiation of cultured marrow stro $^{-}$ mal stem cells on surface of microporous hydroxyapatite based mica composite and macroporous synthetic hydroxyapatite. Biomed Mater Eng 1: 21-26, 1999.

9. Ohgushi H, Caplan AI. Stem cell technology and bioceramics; from cell to gene engineering. J Biomed Mater Res 48: 913-927, 1999.
10. Tamai N, Myoui A, Tomita T et al. Novel hydroxyapatite ceramics with an interconnective porous structure exhibit superior osteoconduction in vivo. J Biomed Mater Res 59: 110-117, 2001.

11. Aubin JE. Bone stem cells. J Cell Biochem Suppl 31: 73-82, 1998.

12. Bruder SP, Jaiswal N, Ricalton NS et al. Mesenchymal stem cells in osteobiology and applied bone regeneration. Clin Orthop Relat Res 355: 247-256, 1998.

13. Langer R, Vacanti JP. Tissue engineering. Science 260: 920-926, 1993.

14. Lieberman JR, Daluiski A, Einhorn TA. The role of growth factors in the repair of bone. Biology and clinical applications. J Bone Joint Surg Am 84: 1032-1044, 2002.

15. Ito Y, Tanaka N, Fujimoto Y et al. Bone formation using novel interconnected porous calcium hydroxyapatite ceramic hybridized with cultured marrow stromal stem cells derived from Green rat. J Biomed Mater Res 69: 454-461, 2004.

16. Tsutsumi S, Shimazu A, Miyazaki K et al. Retension of multilineage differentiation potential of mesenchymal cells during proliferation in response to FGF. Biochem Biophys res Commun 288: 413-419, 2001.

17. Owen M. Marrow stromal stem cells. Cell Sci Suppl 10: 63-76, 1988.

18. Caplan AI. Mesenchymal stem cells. J Orthop Res 9: 641-650, 1991.

19. Pittenger MF, Mackay AM, Beck SC et al. Multilineage potential of adult human mesenchymal stem cells. Science 284: 143-147, 1999.

20. Ayers RA, Simske SJ, Nunes CR et al. Long-term bone ingrowthand residual microhardness of porous block hydroxyapatite implants in humans. J Oral Maxillofac Surg 56: 1297-1301, 1998.

21. Steinkamp JA, Hansen KM, Crissman HA. Flow microfluorometric and light-scattter measurement of nuclear and cytoplasmic size in mammalian cells. J Histochem Cytochem 24: 292-297, 1976.

22. Anderson HC. Molecular biology of matrix vesicles. Clin Orthop 314: 266-280, 1995. 